\title{
Clinical Impact of Pharmaceutical Consultations in Patients Treated for Chronic Obstructive Pulmonary Disease: Study Protocol for a Randomized Controlled Trial (BPCObs study).
}

\section{Djamila Hachemi}

CHU Nimes: Centre Hospitalier Universitaire de Nimes

Géraldine Leguelinel-Blache

CHU Nimes: Centre Hospitalier Universitaire de Nimes

\section{Sophie Bouvet}

CHU Nimes: Centre Hospitalier Universitaire de Nimes

\section{Clarisse Roux-Marson}

CHU Nimes: Centre Hospitalier Universitaire de Nimes

\section{Patrice Ray}

CHU Nimes: Centre Hospitalier Universitaire de Nimes

\section{Nathalie Plouvier}

CHU Nimes: Centre Hospitalier Universitaire de Nimes

\section{Jean-Marie Kinowski}

CHU Nimes: Centre Hospitalier Universitaire de Nimes

Florent Dubois ( $\nabla$ florent.dubois@chu-nimes.fr)

Nimes University Hospital

\section{Study protocol}

Keywords: Chronic obstructive pulmonary disease (COPD), Inhaler devices, Patient education, Pharmaceutical services, Clinical pharmacist, Community pharmacist, Inhalation administration, Medication adherence

Posted Date: June 28th, 2021

DOl: https://doi.org/10.21203/rs.3.rs-486822/v1

License: (c) (i) This work is licensed under a Creative Commons Attribution 4.0 International License. Read Full License 


\section{Abstract}

\section{Background}

Chronic obstructive pulmonary disease (COPD) is an irreversible chronic respiratory disease whose evolution depends on the patient's adherence to inhaler devices. Pharmacists may play a role in adherence to medication therapy in hospital and in primary care interacting with patients to provide advice on proper use. This paper presents the protocol for a randomized controlled trial conducted at a university hospital to assess the clinical impact of pharmaceutical consultations on COPD exacerbations, medical care, adherence to inhaler devices and quality of life.

Methods

This trial will include 226 COPD patients treated with inhaler devices: 94 in a control group with the usual hospital care, 66 receiving a pharmaceutical consultation at the hospital and 66 receiving several pharmaceutical consultations at their community pharmacy. The aim of these interventions is to inform patients about COPD and medication therapy, train them in the proper use of inhaler devices and make them aware of good therapeutic adherence. Patients will be randomized to either the control group or the experimental hospital group by the clinical pharmacist at the hospital. Community pharmacists will include patients in the experimental community group. All patients will be monitored for 12 months by their community pharmacists (CPs). The primary outcome is the mean number of COPD exacerbations. Secondary outcomes include the number of medical consultations, emergency visits and hospitalizations; patients' adherence to inhaler devices and ability to use them and quality of life.

\section{Discussion}

Our study is the first randomized controlled trial in France to assess the effect of pharmaceutical interventions on COPD exacerbations. Study limitations include patient recruitment and the CPs' adherence to follow-up. Indeed, the success of this trial depends on the willingness of CPs to collect the data. However, we envisage this work as the first step towards building a network of CPs trained for clinical research.

Trial registration

Clinicaltrials.gov, NCT03704545. Registered on October $12^{\text {th }}, 2018$. https://clinicaltrials.gov/ct2/show/NCT03704545?cond=COPD\&cntry=FR\&city=nimes\&draw=2\&rank=1

\section{Background}

Chronic obstructive pulmonary disease (COPD) is an irreversible chronic respiratory disease, which can be complicated by exacerbations. According to the World Health Organization, 251 million people worldwide were affected in 2016. Back in 2009, COPD was considered as the fourth leading cause of death in the world and was projected to be the third by 2020. In 2015, COPD prevalence was about $10 \%$ of the adult 
population in France (1). The evolution of the disease depends, among other things, on adherence to medication treatments administered by inhalation devices. Many kinds of devices are available depending on the form of the active substance they contain (capsule, mistletoe, etc.). This means that a patient may use several different devices, successively or simultaneously, depending on the management of his/her pathology. However, the advanced age of patients and the lack of information on the use of inhalation devices are all factors contributing to the misuse of these devices (2). These result in poor adherence to medication estimated at 15 to $50 \%(3)(4)(5)(6)$.

Pharmacists play a leading role in adherence to medication therapy. At the hospital, clinical pharmacists provide patients with advice on how to use their inhalers properly. One French study has shown that a pharmaceutical outpatients consultation improves patients' adherence to medication by $30 \%$ when they return home (7). In primary care, community pharmacists may inform and educate the patient on his/her treatment during each monthly dispensation. This is why pharmaceutical interview programs have been set up to reinforce the follow-up of patients with asthma and patients treated with oral anticoagulants (8) (9)(10)(11). For elderly patients, the pharmacist's role in patient care has proved to have an impact and medication reviews can largely contribute to improving patient safety (12).

Good adherence to inhalation devices seems to slow down the course of COPD. Indeed, a significant decrease in the number of exacerbations has been found in patients who do not make mistakes in taking their treatment (2). A Norwegian study(13) showed that therapeutic education sessions reduced the number of visits to the general practitioner (GP) by $85 \%$ (3.4 versus $0.5, p<0.001)$ and decreased the consumption of short-acting beta2-adrenergic agonists used in the acute phase of the disease by $57 \%$ ( $p$ $<0.03)$. This work also showed a decrease in healthcare costs associated with care management, particularly regarding consultations with GPs. A literature review on community pharmacist intervention highlighted a positive impact on medication adherence and on the patient's ability to use inhaler devices to manage $\operatorname{COPD}(14)$. Also, in Belgium, community pharmacists' interventions increased the rate of adherent patients, reduced the occurrence of severe exacerbations and shortened the length of hospital stays(15).

To our knowledge, pharmaceutical intervention in the management of COPD has not yet been studied in France. Moreover, no studies have explored the potential benefits provided by hospital clinical pharmacists. This trial aims to assess whether pharmaceutical consultations in primary care and/or hospital could have a clinical impact in patients treated for COPD at home. This work is part of a scheme to promote clinical pharmacy activities in France and new missions for community pharmacists focused on improving medication adherence.

\section{Methods / Design}

\section{Design}


Our study is a monocentric randomized controlled trial carried at a French university hospital. The aim of the study is to assess the impact of pharmaceutical consultations on COPD exacerbations after 12 months of follow-up. The secondary objectives are to evaluate the impact on patient care pathway, adherence to inhalation devices and quality of life.

Two types of pharmaceutical intervention will be evaluated (hospital consultations and community consultations) and compared with the usual care strategy provided by a control group. The sudy design is outlined in Fig. 1.

Patients included in hospital will join control or experimental hospital group after randomization. For this study it is impossible to do a 3-armed randomization for feasibility reasons and the risk of a contamination bias. Indeed, to do a 3-armed randomization, patients would all have to be included at the hospital. As it is not possible to impose on patients to pick up their treatment from a selected pharmacy, a strong contamination bias would be present because the pharmacist might find him/herself delivering treatment to two patients randomized to the control and the experimental group. Thus, a significant risk of delivering the same information to patients would be raised. By selecting pharmacies in the experimental community group upstream and including the corresponding patients, this contamination bias is removed. Likewise, patients seen at the hospital will not be included in the study if their usual pharmacy is among the 10 pharmacies taking part in the experimental community group. These pharmacies have already been associated with other projects in connection with the hospital pharmacy department. Obviously, patients included at the hospital will not be included in the study if their usual pharmacy is among the 10 participants in the community experimental group.

The three groups will be matched according to the stage of disease, since the risk of exacerbation or hospitalization increases with the stage, regardless of adherence to medical treatment (16) (17). Because COPD is a chronic, progressive, irreversible disease, patients in the advanced stages are often older, frailer and have more associated co-morbidities. For this criterion, the balance of groups will be checked at the midpoint of inclusions and the following inclusions will be readjusted as necessary.

The study methodology has been overseen by a pilot committee to ensure the relevance of research arrangements and ensure the quality of data collection. The first two committee meetings were held upon acceptance of the project and prior to the start of inclusions. A final meeting will be held when the results are exploited. An audit will be carried out by a clinical research associate during the course of the study.

This protocol has been submitted to the committee for the protection of persons and a request for authorization has been made to the French National Agency for the Safety of Medicines in accordance with the regulations.

\section{Setting and participants}

All patients on inhaler device treatment for Stage 2 to Stage 4 COPD according to the Global Initiative for Chronic Obstructive Lung Disease classification (18) will be eligible. Patients with Stage 1 COPD will not be included as their treatment does not require chronic inhalation therapy. All autonomous patients aged 
over 18 and living at home who have agreed to 12 months of monthly follow-up will be included in the study.

Participants in the hospital group will be hospitalized in care units that benefit from a clinical pharmaceutical activity. Clinical pharmacists will detect eligible patients at admission by medication reconciliation and contact their community pharmacist to ensure their participation throughout follow-up. Recruitment and randomization will be made by the clinical pharmacist as soon as hospital discharge is confirmed. Patients in the community pharmacy group will be included directly by their community pharmacist.

For all groups, an information letter will be presented to the patient, specifying the purpose of the study and how it will be conducted, as well as their right to refuse to participate in the study or leave at any time. Patient consent will be sought and obtained before patients enter the study. One copy of the signed consent will be given to the patient, one will be retained by the investigator, and one will be retained by the sponsor.

The study visits, procedures and assessments are outlined in the Tables $1 \mathrm{a}$ and $1 \mathrm{~b}$. An additional file shows The Standard Protocol Items: Recommendations for Interventional Trials (SPIRIT) checklist [see additional file 1].

\section{Outcomes}

\section{Primary outcome:}

The primary outcome will assess the mean number of COPD exacerbations by patient after 12 months of follow-up. Exacerbations are defined as "periods of increased COPD symptoms (dyspnea, cough, sputum) requiring consultation with a GP, pulmonologist or hospitalization". The study coordinating pharmacist will phone the patient's GP or pulmonologist at 3, 6, 9 and 12 months of follow-up to collect the results.

\section{Secondary outcomes:}

Among the secondary outcomes, four measures will be collected by the same method as for the primary outcome mentioned above: [1] number of hospitalizations, [2] number of emergency visits, [3] number of visits to the GP and [4] number of visits to the pulmonologist. Other secondary outcome measures will be collected by the patient's community pharmacist using a specific data collection book: [5] adherence to medication, evaluated by calculating the Medication Possession Ratio. This is the ratio of the actual number of doses/capsules remaining compared with the theoretical number of doses/capsules remaining (i.e. the difference between the number of doses/capsules dispensed compared with the number prescribed) (19); [6] the number of completed successive steps common to all inhaler devices (i.e. slowly exhaling, inhaling and then holding the breath for 5 seconds) (20); [7] the patients' quality of life via the BPCO-VQ11 self-questionnaire which is specific to COPD (21) collected at inclusion and then 6 and 12 months of follow-up. 


\section{Intervention}

The flow of the intervention is outlined in Fig. 2.

Experimental hospital group intervention

Patients in the experimental hospital group will benefit from a Hospital Pharmaceutical Consultation (HPC). At the end of hospitalization, the HPC will be carried out by a clinical hospital pharmacist trained by the study-coordinating pharmacist, using a consultation guideline, in order to harmonize practices. This guideline was written by two clinical pharmacists and validated by two pulmonologists from the hospital. During the HPC lasting approximately 20 minutes long, the patient will be informed about his/her disease and the treatment principles (i.e. how it works, adverse effects and how to use the inhaler by means of a demonstration with placebo inhalers). If necessary, the pharmacist will sensitize patients to the importance of giving up smoking as the disease progresses. The aim of this consultation is to explain the importance of good adherence and answer any questions the patient may have. The tools used will be standardized information supports created specifically for the project by two clinical pharmacists and validated by two pulmonologists from the hospital.

Experimental community group intervention

Patients in the community group will have an Initial Community Pharmacy Consultation (ICC) and, if required, several monthly Follow-up Community Pharmacy Consultations (FCCs) with their community pharmacist. The study-coordinating pharmacist will have trained these pharmacists beforehand during a meeting to brief them on both types of consultation. The consultation guidelines have been written and validated by the same people as those who validated the HPC.

\section{a) Initial Community Pharmacy Consultation}

The ICC will be performed during the course of COPD treatment at the patient's inclusion visit. The ICC will contain the same information as the HPC. The tools used will be the same, standardized information supports as those created and used for the HPC. Demonstration placebo inhalers provided by the sponsoring hospital center will also be used during this consultation.

\section{b) Follow-up Community Pharmacy Consultations}

At the 11 consultations following the ICC at which inhaler devices are dispensed, the community pharmacist will check the patient's adherence and ability to use the devices. If he/she finds any device misuse, he/she will give a FCC lasting approximately 10 minutes, consisting of a new demonstration of how to use the inhaler and a reminder of the information given at the ICC. In all cases, the pharmacist will answer any questions the patient may have. The tools used are the same as those used for the ICC.

Patients in the control group will benefit from the usual practice without ICC and FCCs. All patients included in the study will be followed up monthly by their community pharmacist to collect some outcomes. 


\section{Blinding}

Because of the nature of the interventions, blinding will not be possible in our study for patients and care providers. Therefore, this study is fully open, without reliable blinding.

\section{Sample size calculation}

This study will compare three arms. A control group will be compared with two experimental groups: one group with a pharmaceutical consultation at the hospital and one group with several pharmaceutical consultations at their community pharmacy. According to the literature(15), the average number of exacerbations is 0.61 per patient per year. If we make the hypothesis of a $20 \%$ minimum decrease with one of the two experimental arms at a $5 \%$ alpha threshold and a statistical power of $90 \%$, we obtain 60 patients per group. Since the control group will be used for two comparisons, its size was increased by a root factor, i.e. 85 patients. Considering $10 \%$ of patients will be lost to follow-up, a total of 226 patients will be enrolled in the study: 94 in the control group and 66 in each intervention group (hospital and community pharmacy) (Fig. 1).

\section{Data collection}

Only those involved in the research project and identified will have access to the RedCap ${ }^{\circledR}$ data entry software. All data entered in the electronic case report form will be controlled and formatted to prevent the entry of out of bounds data or outliers. In the event of an input change, traceability will be ensured. This software is hosted on our University Hospital's website and access to the application is protected by a login and password. All data collected via this software are backed up daily on a secure network.

All clinical data from the study will be stored on a specific server directory. Only network administrators and authorized persons in the Department of Biostatistics, Epidemiology, Public Health and Innovation in Methodology (BESPIM) will have access to this directory.

A clinical research assistant delegated by the promoter will regularly monitor the study in accordance with the regulations in force: at the outset, during the study and at the end. The frequency of visits will depend on the inclusion rate. It will aim to monitor compliance with the protocol, verify informed consent, ensure quality control and alert to any possible deviations from the protocol. All visits will be the subject of a monitoring report in the form of a written report (traceability of visits).

\section{Data analysis}

The average number of hospitalized patients at our institution on treatment for COPD and eligible for management by a clinical pharmacist is estimated at 1200 per year. Recruitment is planned for over a period of 24 months. As COPD is a high-prevalence disease, estimated at $10 \%$ in the adult population in France in 2016 (1), patient recruitment by selected community pharmacies will be achievable during the inclusion period. 


\section{Description of the population and main parameters under study}

An initial data analysis will allow us to describe the total population and by group (control vs. intervention). Statistical results will be presented as means \pm standard deviations for quantitative variables with Gaussian distribution, and medians and interquartile ranges for other variables. For qualitative variables, the numbers and associated percentages will be presented.

\section{Statistical analysis}

The statistical analysis will be conducted by the BESPIM at Nîmes University Hospital using statistical analysis software (SAS Institute, Cary, NC, USA) version 9 or R 3.5.0.

All analyses will be made according the intention-to-treat principle and all statistical tests will be conducted at 0.05 two-sided significance level.

\section{a) Initial comparability of groups}

No statistical comparison between groups will be made for either randomized group. The experimental community, which is matched on disease stage with the experimental hospital group, will be compared with the other two groups: qualitative variables will be compared by a Chi-squared or Fisher's exact test depending on conditions. ANOVA or Kruskal-Wallis test will be used for the quantitative variables.

\section{b) Analysis of primary outcome measures}

The mean number of exacerbations will be compared for the three groups using a Kruskal-Wallis test to assess the overall differences between them at 12 months. The two experimental groups will then be compared with the control group using a Student T-test or Mann-Whitney-Wilcoxon test according to distribution.

A subgroup analysis is also planned: The mean number of exacerbations will be estimated according to severity groups and compared via a Kruskal-Wallis test for all three groups. The rate of patients with at least one exacerbation will be compared for the three groups using a Chi-squared test.

\section{c) Analysis of secondary outcome measures}

The criteria for the number of consultations, emergency room visits and hospitalizations will be estimated and compared using a Kruskal-Wallis test. Then the two experimental groups will be compared with the control group using a Student T-test or Wilcoxon-Mann-Whitney test according to the distribution.

The medication Possession Ratio and the number of completed successive steps common to all inhalation devices will be described per group and each month. A graphical analysis of the evolution will be made. 
Furthermore, the median Medication Possession Ratio and the median number of completed successive steps common to all inhalation devices estimated will be compared per group using a Kruskal-Wallis test.

The evolution of quality of life over time will be described via a graphical analysis. The average scores for the three groups will also be presented and compared.

\section{Dissemination}

The corresponding author will be responsible for the publication of the results of the study as well as any publications ancillary to the project. No intermediate publication of results will be made.

\section{Discussion}

We hereby describe the protocol for a clinical trial designed to evaluate the impact of pharmaceutical consultations at the hospital or community pharmacy on the occurrence of COPD exacerbations in patients using inhaler devices. To our knowledge, this is the first randomized controlled trial to evaluate the effects of this kind of intervention in France. Due to the nature of the intervention, blinding will not be possible for patients or pharmacists. Therefore, this study is fully open, without reliable blinding.

Two critical parameters will be taken into account to guarantee the study's feasibility: patient recruitment and availability for follow-up. The first parameter may be explained by the fact that patients with COPD are elderly, frail patients, generally suffering from several comorbidities. Even though the number of hospitalized COPD patients is high, the proportion of eligible subjects available for a 12-month follow-up upon their return home is reduced. There are several reasons for this: the life expectancy of certain patients, the transfer to follow-up care and the intervention of nurses at home. These patients' characteristics also represent a risk of them becoming lost to follow-up.

Concerning outcomes, there is a potential information bias due to the method used for collecting data on exacerbations, physician (GP and pulmonologist) visits, hospitalization and emergency visits. Indeed, the exhaustiveness of data collection by telephone call is limited as this depends on the physicians' availability. To harmonize data collection, the study-coordinating pharmacist will centralize all telephone calls.

To standardize the information provided during consultations, pharmacists have been trained by the study-coordinating pharmacist using guidelines developed in collaboration with the pharmacy and pulmonology teams. At each training session, pharmacists for the two experimental groups (community and hospital) were able to ask questions about the study and follow-up. For patients included at hospital (control and experimental hospital groups), their community pharmacist will only have to monitor and collect data without any intervention. There is a risk of bias due to potential information given to the patient by the pharmacist during dispensing.

Lastly, another limitation will be the community pharmacist's adherence to follow-up including control group and experimental hospital group pharmacists. Indeed, the willingness of these pharmacists and the 
team's availability to collect data will have an impact on the quality and quantity of data collected. Throughout the study, the study-coordinating pharmacist will provide follow-up and telephone assistance as required. However, this study could be the first stage towards building a network of community pharmacists trained in clinical research. With this in mind, we would like to provide a model for future studies in which patients can be monitored over long periods with real-life data collected by their community pharmacists.

\section{TRIAL STATUS}

This trial was registered on October 12th, 2018 in Clinicaltrials.gov under the number NCT03704545. Actually, 149 patients on 226 have been included since 18 January 2019. Recruitment should be completed by July 2021 .

\section{Abbreviations}

BESPIM

Department of Biostatistics, Epidemiology, Public Health and Innovation in Methodology; COPD:Chronic obstructive pulmonary disease; CP:Community Pharmacists; FCC:Follow-up Community Pharmacy Consultations; GP:General Practitioner; HPC:Hospital Pharmacy Consultation; ICC:Initial Community Pharmacy Consultations; SPIRIT:Standard Protocol Items:Recommendations for Interventional Trials.

\section{Declarations}

\section{Ethics approval and consent to participate}

This study will be performed in accordance with the Declaration of Helsinki and has been approved by the committee for the protection of persons, CPP Sud Méditerranée III; reference no. 2018.10.01 six_18.07.09.52123) and by the French National Agency for the Safety of Medicines (ANSM; reference no. 2018-A01699-46). Written informed consent will be sought from all patients included.

\section{Consent for publication}

Not applicable

\section{Availability of data and materials}

All datasets generated from the study are available from the corresponding author.

\section{Competing interests}

The authors declare that they have no competing interests.

\section{Funding}


This trial is supported by Nimes University Hospital internal funding through the NimAO 2017 call for tenders. The study protocol has undergone peer-review by the funding body. The funders have a role on this study in protocol drafting, data collection, data analysis and interpretation.

\section{Authors' contributions}

DH: developing consultation guidelines and supports for the interventions, member of the pilot committee, drafting the initial manuscript. GLB: co-writing and correcting the protocol, co-investigator, member of the pilot committee. SB: writing the case report form, performing statistical analysis, member of the pilot committee. CRM: co-writing and correcting the protocol, co-investigator. NP: co-writing and correcting the protocol, validating consultations guidelines and supports for interventions, co-investigator, member of the pilot committee. PR: co-writing and correcting the protocol, validating consultations guidelines and supports for interventions, co-investigator. JMK: co-writing and correcting the protocol, principal investigator, member of the pilot committee. FD: writing the protocol, submitting the protocol to the funding tender, planning the study, developing consultation guidelines and supports for the interventions, co-investigator, member of the pilot committee, drafting the initial manuscript. All authors have read and approved the final manuscript.

\section{Acknowledgements}

The authors thank the Occitania regional union of health professionals and all community pharmacists for making this study possible. The authors also wish to thank the Department of Biostatistics, Epidemiology, Public Health and Innovation in Methodology (BESPIM) for their support. Particular thanks go to Teresa Sawyers, English Medical Writer at the BESPIM, for her help with editing this paper.

\section{Author details}

a Department of Pharmacy, CHU Nimes, Univ Montpellier, Nimes, France ; b UPRES EA2415, Laboratory of Biostatistics, Epidemiology, Clinical Research and Health Economics, Clinical Research University Institute, Univ Montpellier, Montpellier, France ; ' Department of Law and Health Economics, Univ Montpellier, Montpellier, France ; ${ }^{d}$ Department of Biostatistics, Epidemiology, Public Health and Innovation in Methodology, CHU Nimes, Univ Montpellier, Nimes, France ; ${ }^{e}$ Department of Pulmonology, CHU Nimes, Univ Montpellier, Nimes, France

\section{References}

1. Santé Publique France. Connaissance de la bronchopneumopathie chronique obstructive (BPCO) en France: Baromètre santé 2017 [Internet]. [cité 15 mai 2020]. Disponible sur:

https://www.santepubliquefrance.fr/maladies-et-traumatismes/maladies-et-infectionsrespiratoires/bpco-et-insuffisance-respiratoire-chronique/documents/article/connaissance-de-labronchopneumopathie-chronique-obstructive-bpco-en-france-barometre-sante-2017. 
2. Molimard M, Raherison C, Lignot S, Balestra A, Lamarque S, Chartier A, et al. Chronic obstructive pulmonary disease exacerbation and inhaler device handling: real-life assessment of 2935 patients. Eur Respir J. 1 févr 2017;49(2).

3. Dolce JJ, Crisp C, Manzella B, Richards JM, Hardin JM, Bailey WC. Medication adherence patterns in chronic obstructive pulmonary disease. Chest avr. 1991;99(4):837-41.

4. Rolnick SJ, Pawloski PA, Hedblom BD, Asche SE, Bruzek RJ. Patient characteristics associated with medication adherence. Clin Med Res juin. 2013;11(2):54-65.

5. Haupt D, Krigsman K, Nilsson JLG. Medication persistence among patients with asthma/COPD drugs. Pharm World Sci PWS oct. 2008;30(5):509-14.

6. Mueller S, Wilke T, Bechtel B, Punekar YS, Mitzner K, Virchow JC. Non-persistence and non-adherence to long-acting COPD medication therapy: A retrospective cohort study based on a large German claims dataset. Respir Med. 2017;122:1-11.

7. Leguelinel-Blache G, Dubois F, Bouvet S, Roux-Marson C, Arnaud F, Castelli C, et al. Improving Patient's Primary Medication Adherence: The Value of Pharmaceutical Counseling. Medicine (Baltimore) oct. 2015;94(41):e1805.

8. Ministère des affaires sociales et de la santé. Arrêté du 24 juin 2013 portant approbation de l'avenant $\mathrm{n}^{\circ} 1$ à la convention nationale du 4 avril 2012 organisant les rapports entre les pharmaciens titulaires d'offıcine et l'assurance maladie relatif à l'accompagnement des patients chroniques sous anticoagulants oraux.

9. Arrêté du 28. novembre 2014 portant approbation des avenants nos 3, 4 et 5 à la convention nationale du 4 mai 2012 organisant les rapports entres les pharmaciens titulaires d'officine et l'assurance maladie.

10. Arrêté. du 4 mai 2012 portant approbation de la convention nationale organisant les rapports entre les pharmaciens titulaires d'officine et l'assurance maladie.

11. Arrêté. du 9 mars 2018 portant approbation de l'avenant 12 à la convention nationale du 4 mai 2012, organisant les rapports entres les pharmaciens titulaires d'officine et l'assurance maladie.

12. Tasai $\mathrm{S}$, et al. Impact of Medication Reviews Delivered by Community Pharmacist to Elderly Patients on Polypharmacy: A Meta-analysis of Randomized Controlled Trials [Internet]. Journal of patient safety. J Patient Saf; 2019 [cité 20 mai 2020]. Disponible sur: https://pubmed.ncbi.nlm.nih.gov/30920431/.

13. Gallefoss $F$. The effects of patient education in COPD in a 1-year follow-up randomised, controlled trial. Patient Educ Couns mars. 2004;52(3):259-66.

14. Hesso I, Gebara SN, Kayyali R. Impact of community pharmacists in COPD management: Inhalation technique and medication adherence. Respir Med. 2016;118:22-30.

15. Tommelein E, Mehuys E, Van Hees T, Adriaens E, Van Bortel L, Christiaens T, et al. Effectiveness of pharmaceutical care for patients with chronic obstructive pulmonary disease (PHARMACOP): a randomized controlled trial. Br J Clin Pharmacol mai. 2014;77(5):756-66. 
16. Hurst JR, Vestbo J, Anzueto A, Locantore N, Müllerova H, Tal-Singer R, et al. Susceptibility to Exacerbation in Chronic Obstructive Pulmonary Disease. N Engl J Med. sept 2010;16(12):1128-38. 363(.

17. Donaldson GC. COPD exacerbations: Epidemiology. Thorax 1 févr. 2006;61(2):164-8.

18. Cf, Vogelmeier, et al. V. Global Strategy for the Diagnosis, Management, and Prevention of Chronic Obstructive Lung Disease 2017 Report. GOLD Executive Summary [Internet]. Vol. 195, American journal of respiratory and critical care medicine. Am J Respir Crit Care Med; 2017 [cité 20 mai 2020]. Disponible sur: https://pubmed.ncbi.nlm.nih.gov/28128970/?from_term=gold+copd\&from_pos=2.

19. Cramer JA, Roy A, Burrell A, Fairchild CJ, Fuldeore MJ, Ollendorf DA, et al. Medication compliance and persistence: terminology and definitions. Value Health J Int Soc Pharmacoeconomics Outcomes Res févr. 2008;11(1):44-7.

20. Assurance Maladie. Traitement de la BPCO [Internet]. [cité 15 mai 2020]. Disponible sur: https://www.ameli.fr/assure/sante/themes/bpco-bronchite-chronique/traitement.

21. Ninot G, Soyez F, Fiocco S, Nassih K, Morin AJS, Prefaut C. [The VQ11, a short health-related quality of life questionnaire for routine practice in COPD patients]. Rev Mal Respir mai. 2010;27(5):472-81.

\section{Tables}

Table 1a: Visits, chronology and procedures for the Control and Experimental Hospital groups. 


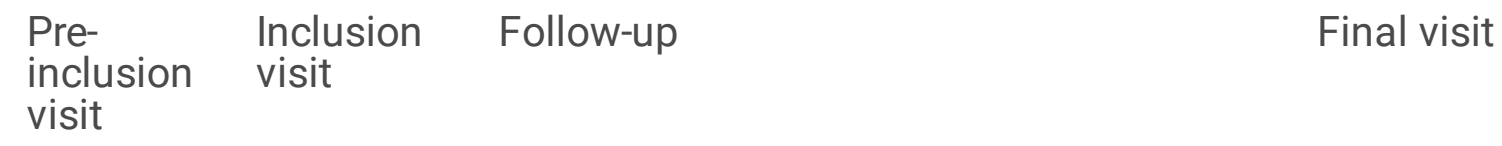

\begin{tabular}{|c|c|c|c|c|c|c|}
\hline EVENT & $\begin{array}{l}\text { Hospital } \\
\text { stay }\end{array}$ & $\begin{array}{l}\text { Hospital } \\
\text { discharge }\end{array}$ & $\begin{array}{l}\text { First } \\
\text { dispensing } \\
\text { by the CP } \\
\text { (Dispensing } \\
\text { 1) }\end{array}$ & $\begin{array}{l}\text { Dispensing } \\
2 \text { to } \\
\text { Dispensing } \\
11\end{array}$ & $\begin{array}{l}\text { Dispensing } \\
12\end{array}$ & $\begin{array}{l}\text { Dispensing } \\
12\end{array}$ \\
\hline
\end{tabular}

\section{ENROLMENT}

\begin{tabular}{ll}
$\begin{array}{l}\text { General } \\
\text { information }\end{array}$ & $\mathrm{x}$ \\
$\begin{array}{l}\text { Presentation of } \\
\text { the briefing note }\end{array}$ & $\mathrm{x}$ \\
$\begin{array}{l}\text { Validation of } \\
\text { inclusion and } \\
\text { non-inclusion } \\
\text { criteria }\end{array}$ & $\mathrm{x}$ \\
\hline
\end{tabular}

Collection of

informed

$x$

consent Randomization $\quad x$

\section{INTERVENTION}

HPC ( hospital

experimental

group)

\section{EVALUATION}

\section{$\mathrm{x}$}

(Dispensing

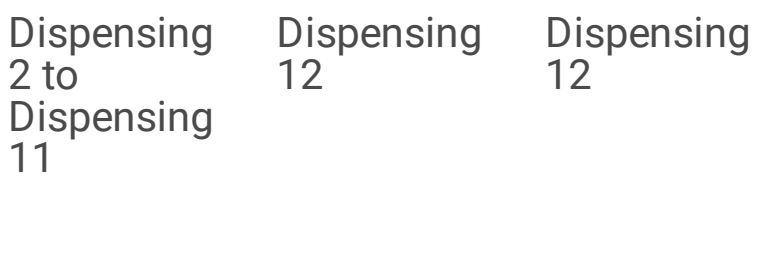

Collection of remaining

doses/capsules

Ability score to inhalation device

X $\quad x$

x $\quad x$

Collection of exacerbations, consultations, hospitalizations, visits to the emergency service

\section{BPCO VQ11}

Questionnaire 
Table 1b. Visits, chronology and procedures for the Experimental Community group.

\begin{tabular}{|c|c|c|c|c|c|}
\hline & $\begin{array}{l}\text { Pre- } \\
\text { inclusion } \\
\text { visit }\end{array}$ & $\begin{array}{l}\text { Inclusion } \\
\text { visit }\end{array}$ & Follow-up & & Final visit \\
\hline EVENT & $\begin{array}{l}\text { Dispensing } \\
\text { prior to the } \\
\text { inclusion } \\
\text { visit }\end{array}$ & $\begin{array}{l}\text { First } \\
\text { dispensing } \\
\\
\text { by the CP } \\
\text { (Dispensing } \\
\text { 1) }\end{array}$ & $\begin{array}{l}\text { Dispensing } \\
2 \text { to } \\
\text { Dispensing } \\
11\end{array}$ & $\begin{array}{l}\text { Dispensing } \\
12\end{array}$ & $\begin{array}{l}\text { Dispensing } \\
12\end{array}$ \\
\hline
\end{tabular}

\section{ENROLMENT}

General information $\quad x$

Presentation of the $\quad x$

briefing note

Validation of inclusion $\quad \mathrm{x}$

and non-inclusion criteria

Collection of informed

consent

$x$

\section{INTERVENTION}

ICC

$\mathrm{X}$

FCC

\section{EVALUATION}

Collection of remaining doses/capsules

Ability score to inhalation device

Collection of exacerbations, consultations, hospitalizations, visits to the emergency

\section{BPCO VQ11}

Questionnaire

$x$
$x$

(Dispensing

1)

x




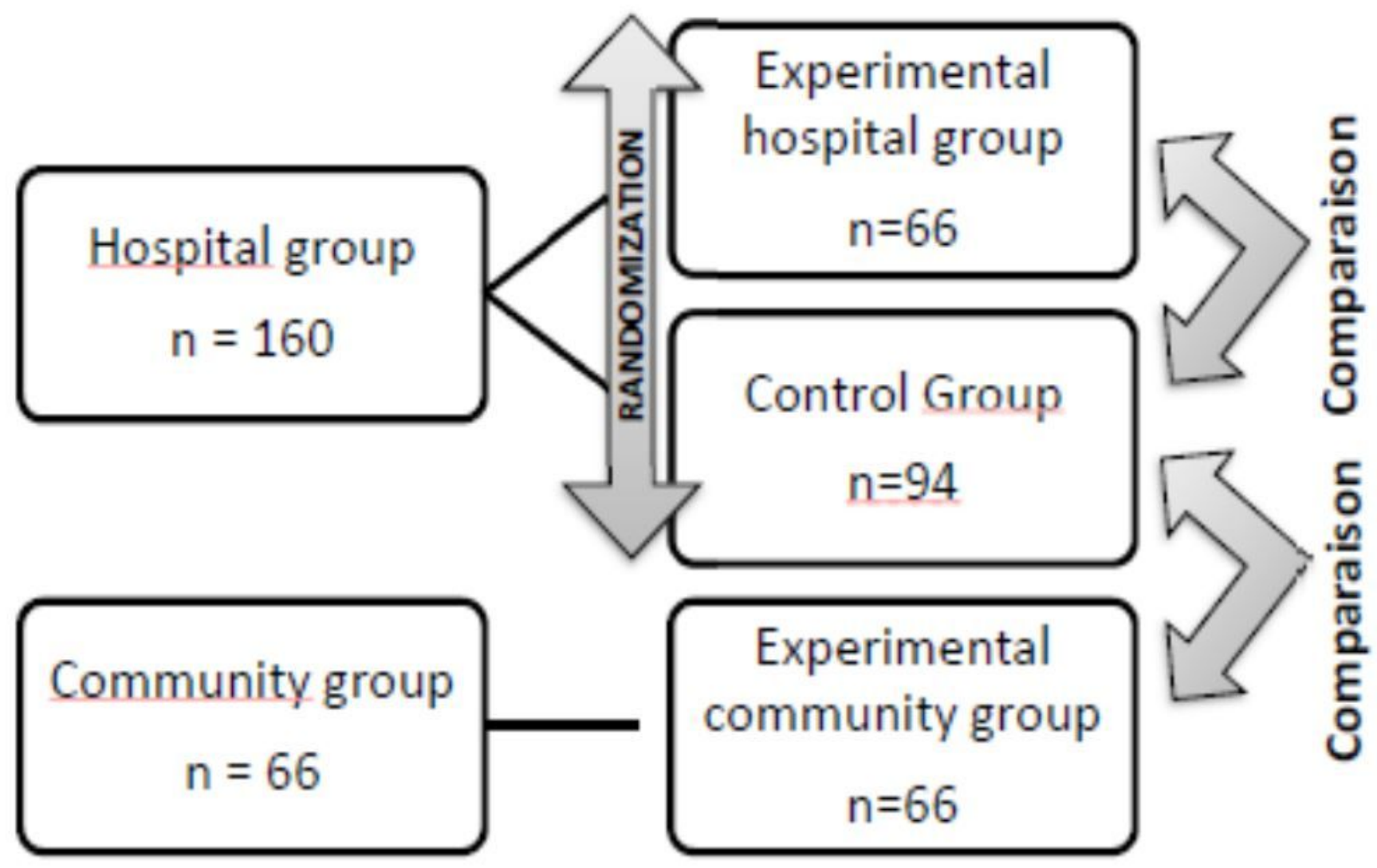

Figure 1

BPCObs Study Design 


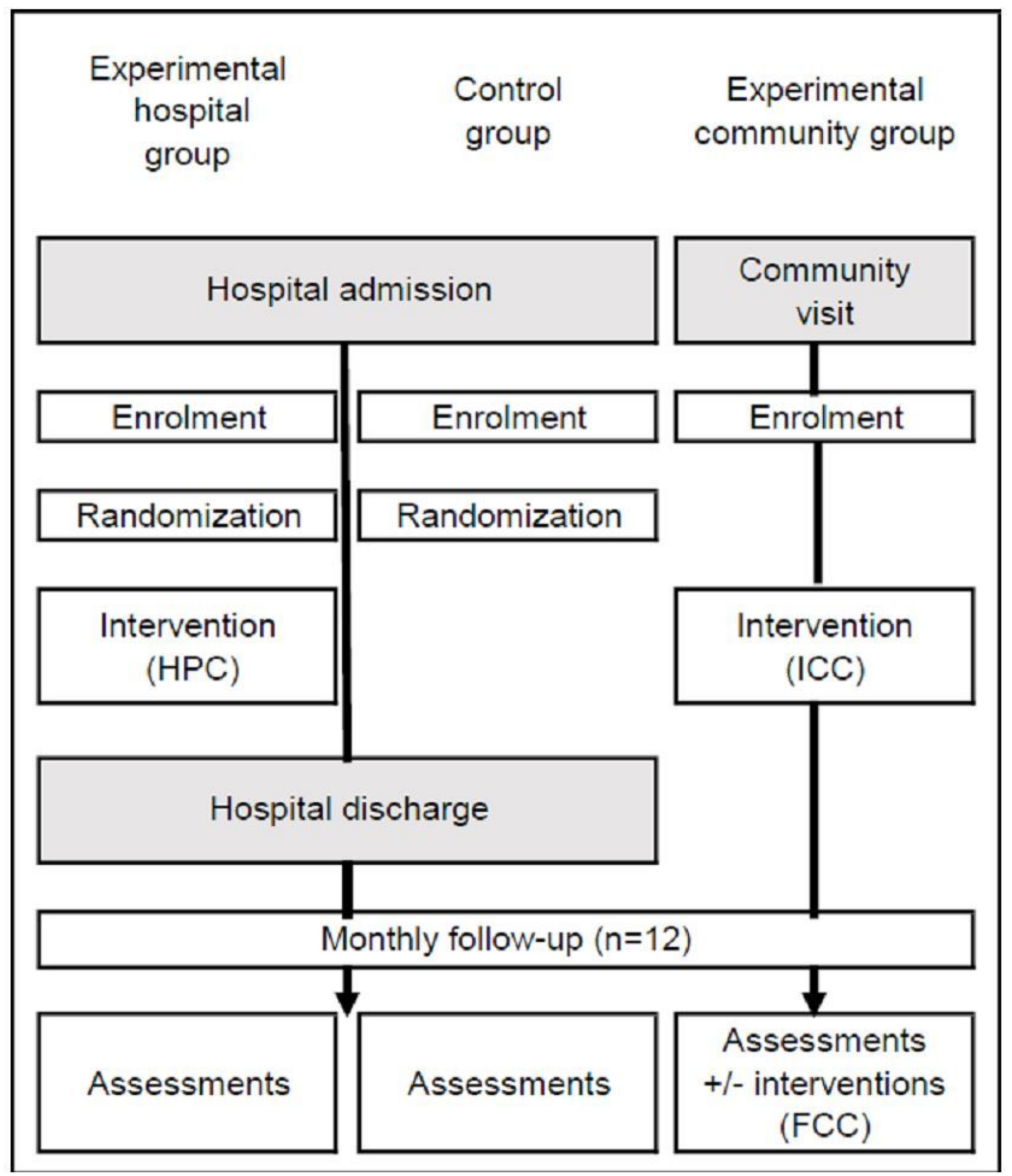

Figure 2

BPCObs study flowchart

\section{Supplementary Files}

This is a list of supplementary files associated with this preprint. Click to download. 
- Annex1Freeandinformedconsentform.docx

- BPCOBSstudyprotocol.pdf 